\title{
Cirurgia de revascularização do miocárdio sem o emprego de soluções cardioplégicas, balão intra-aórtico, ou catéter de Swan-Ganz
}

\author{
E. Régis JUCÁ*, Waldemiro CARVALHO Jr. ${ }^{\star}$, Ciro CIARLINI*, Fernando MESQUITA*, Leny MONTE*, Maria \\ Goretti A. ALVES*
}

RBCCV 44205-45

\begin{abstract}
JUCÁ, E. R.; CARVALHO Jr., W.; CIARLINI, C.; MESQUITA, F.; MONTE, L.; ALVES, M. G. A. - Cirurgia de revascularização do miocárdio sem o emprego de soluções cardioplégicas, balāo intra-aórtico, ou catéter de Swan-Ganz. Rev. Bras. Cir. Cardiovasc., 3(1):21-28, 1988.

RESUMO: No período de 1974 a 1987, 620 pacientes foram submetidos a cirurgia de revascularização do miocárdio, na Casa de Saúde São Raimundo, com 28 óbitos, 4,5\% de mortalidade. Dezenove pacientes foram submetidos a procedimentos associados: prótese aórtica, mitral, ou ressecção de aneurisma ventricular. Os pacientes com idade acima de 70 anos e os submetidos a reoperação estão incluídos. Nāo houve seleção dos casos. $O$ método de proteção miocárdica foi o do pinçamento intermitente da aorta, hipotermia corporal de $30^{\circ} \mathrm{C}$ e hipotermia tópica com solução salina gelada. A síndrome de baixo débito, a mediastinite em paciente diabético, anemia pré-operatória em paciente com angina instável acima de 70 anos de idade, tamponamento cardíaco tardio, endarterectomia não satisfatória, desatenção no uso de nipride no pós-operatório, choque após a protamina, sangramento, acidente vascular cerebral, coma hiperosmolar foram, entre outras, as causas de óbito. Da presente experiência, concluímos que o pinçamento intermitente da aorta, associado à hipotermia, é um aceitável método de preservação do miocárdio.
\end{abstract}

DESCRITORES: miocárdio, revascularização, cirurgia; miocárdio, preservação.

\section{INTRODUÇÃO}

A cirurgia de revascularização do miocárdio exige. para perfeiçâo da anastomose, um campo exangüe e relativamente imóvel, Isto pode ser obtido com a ințerrupção do fluxo coronário, por pinçamento da aorta ascendente, por oclusão da artéria coronária ${ }^{1-4},{ }^{11}$.

O pinçamento da aorta ascendente induz a parada cardiaca por anóxia. A anóxia por longos períodos de tempo é deletéria e exige métodos de proteção miocárdica, como hipotermia corporal, hipotermia tópica exter- na, ou, mesmo, intracoronária. Esta última é sempre feita através de solução cardioplégica gelada contendo potássio em diferentes concentraçōes ${ }^{8}$ 11, 12 . É fora de dúvida que o uso de soluçāo cardioplégica gelada favorece os resulados da cirurgia cardíaca. Entretanto, um número elevado de questōes é levantado, a partir da pergunta inicial: qual a melhor soluçāo: sangüinea, ou cristalóide; qual a melhor concentração de $\mathrm{K}^{+}$, osmolaridade, $\mathrm{pH}$, quantidade e pressão da infusão, drogas (manitol, nifedipina, xilocaina, cortisona e outras), sem falar da distribuiçāo desigual no miocárdio, devido às estenoses das artérias coronárias ${ }^{1,9}$. 
JUCÁ, E. R.; CARVALHO Jr., W.; CIARLINI, C.; MESQUITA, F.; MONTE, L.; ALVES, M. G. A. - Cirurgia de revascularizaçāo do miocárdio sem o emprego de soluçōes cardioplégicas, balāo intra-aórtico, ou catéter de Swan-Ganz. Rev. Bras. Cir. Cardiovasc., 3(1): 21-28, 1988.

Ao uso do catéter de Swan-Ganz e do balão intraaórtico, são, também, creditados os melhores resultados da cirurgia cardiaca em nossos dias ${ }^{10,11}$.

Em outubro de 1972, iniciamos a cirurgia de revascularização do miocárdio, em Fortaleza, no Hospital de Messejana, e, até o presente momento, temos usado o pinçamento intermitente, com episódios de cardioplegia isquêmica intercalados por periodos de reperfusāo, associados a hipotermia tópica e corporal, como método de proteção do miocárdio.

No presente trabalho, analisamos os nossos resultados em cirurgia de revascularização do miocárdio sem o uso de solução cardioplégica, balāo intra-aórtico, ou catéter de Swan-Ganz, em 620 pacientes operados na Casa de Saúde São Raimundo, entre 1974 e 1987. O critério para análise da experiência somente na Casa de Saúde São Raimundo, foi a maior facilidade de revisāo.

\section{CASUÍSTICA E MÉTODO}

\section{Casuistica}

No período de 1974 a 1987 , foram submetidos a revascularização do miocárdio 620 pacientes, na Casa de Saúde São Raimundo, em Fortaleza (Tabela 1). A idade média foi de $57,7 \% \pm 8,2$, tendo o mais jovem 36 anos e o mais velho 76 (excluída uma paciente de 9 anos de idade, com endocardite bacteriana, que recebeu uma prótese aórtica e revascularização do miocárdio com ponte de safena para o ramo descendente anterior da coronária esquerda (DA).

Seis por cento dos pacientes estavam acima de 70 anos de idade e $81 \%$ eram do sexo masculino. $O$ número de pontes variou de 1,9 a $2,2 \%$ e a 2,46 por paciente, conforme o periodo. Empregamos pontes seqüenciais em $29 \%$ dos pacientes (Tabela 2).

A mamária interna esquerda foi usada em pacientes abaixo de 60 anos, quando seu diâmetro fosse maior do que o DA e em pacientes que não apresentassem insuficiência coronária aguda, ou hipertrofia ventricular

TABELA 1

CIRURGIA DE REVASCULARIZACCĀO DO MIOCÁRDIO 1974-1987

\begin{tabular}{lrcr}
\hline & N. & MORTAL. & $\%$ \\
\hline R.M. isolada & 601 & 25 & 4,3 \\
R.M. + Proc. Assoc. & 19 & 3 & 15,7 \\
\hline & 620 & 28 & 4,5 \\
\hline
\end{tabular}

R.M. = Revascularização do miocárdio

Proc. Assoc. $=$ Procedimentos associados esquerda. Em 4 pacientes, foi usado um enxerto de mamária bovina conservada em glutaraldeído.

TABELA 2

REVASCULARIZACYAOO DO MIOCARDIO NÚMERO DE PONTES

1987

\begin{tabular}{ccc}
\hline N:PONTES & N: & TOTAL \\
\hline 1 & 12 & 12 \\
2 & 54 & 108 \\
3 & 33 & 99 \\
4 & 02 & 08 \\
\hline
\end{tabular}

N. DE PONTES/PT $=2,20$

PONTE SEQÜENCIAL $=29 \%$

Dezenove pacientes receberam procedimentos associados: prótese aórtica 3, prótese mitral 2, aneurismectomia ventricular 14 .

A maioria dos pacientes apresentava-se hemodinamicamente estável; 28 haviam recebido estreptoquinase. Alguns apresentavam infarto do miocárdio em evolução, mas sem baixo débito cardiaco.

\section{Método}

A monitorização do paciente consistiu somente nas medidas de pressão venosa central através de veia do braço, da pressão arterial (PA) através de cateterização da artéria radial em manômetro de mercúrio, do débito urinário, do eletrocardiograma (ECG) e da temperatura esôfago-faríngea e da pressão de átrio esquerdo na saída de perfusão até o momento da síntese torácica.

A veìa safena foi retirada a partir da prega ingüinal e a mamária esquerda, dissecada e exposta com um afastador de Finochieto pequeno e um afastador do tipo alavanca, idealizado por Jesse Teixeira.

Desde 1979, usamos uma cânula de 2 estágios no átrio direito, para drenagem venosa, e o retorno arterial, feito através da aorta ascendente. Os oxigenadores foram os de bolhas, com permutador de calor integrado, de fabricação nacional.

As anastomoses distais foram feitas por sutura contínua com Prolene 7.0, com 1 ou 2 fios e os nós na lateral e sempre com pinçamen o da aorta ascendente.

O tempo médio do primeiro clampeamento foi de 20,56 minutos $\pm(7,58)$, o do segundo, de $18,20 \pm$ $(5,63)$ e o do terceiro, de $15,52 \pm(4,58)$ e a temperatura média foi de $30^{\circ} \mathrm{C}\left(28\right.$ a $\left.32^{\circ} \mathrm{C}\right)$. O tempo médio de pinçamento total da aorta foi de 31,51 minutos (Tabela 3). 
JUCÁ, E. R.; CARVALHO Jr., W.; CIARLINI, C.; MESQUITA, F.; MONTE, L.; ALVES, M. G. A. - Cirurgia de revascularização do miocárdio sem o emprego de soluçōes cardioplégicas, balão intra-aórtico, ou catéter de Swan-Ganz. Rev. Bras. Cir. Cardiovasc., 3(1): 21-28, 1988.

\begin{tabular}{lc} 
TABELA 3 \\
$\begin{array}{c}\text { REVASCULARIZAÇAO DO MIOCARDIO } \\
\text { PINÇAMENTO INTERMITENTE }\end{array}$ \\
\hline & TEMPO $(\mathrm{min})$ \\
\hline & $20,56 \pm(7,58)$ \\
1: PINÇAMENTO & $18,20 \pm(5,63)$ \\
2. PINÇAMENTO & $15,52 \pm(4,58)$ \\
3: PINÇAMENTO & \\
TEMPO MÉDIO TOTAL & $31,51 \mathrm{~min} \pm(11,86)$ \\
TEMP CORP. MÉDIA & $=32^{\circ} \mathrm{C} \pm(1,97)$ \\
TEMP. MINIMA & $=28^{\circ} \mathrm{C}$ \\
\hline
\end{tabular}

A anastomose proximal, em $90 \%$ dos casos, foi realizada com pinçamento lateral da aorta e com o coração batendo, ou fibrilando, no período de reaquecimento. Em casos de reoperação, ou aorta calcificada, a anastomose proximal foi realizada com pinçamento total e descompressão da aorta ascendente.

Não usamos drenagem (venting) do átrio esquerdo, ou do ventrículo esquerdo, e somente na aorta ascendente, nos períodos de cardioplegia isquêmica, para realização das anastomoses distais.

O orificio na aorta ascendente foi utilizado para realização de uma das anastomoses proximais. Entre uma e outra, ou entre duas e outra anastomose distal, realizamos reperfusão por despinçamento da aorta. À hipotermia corporal de $30^{\circ} \mathrm{C}$, somamos a hipotermia tópica com solução salina gelada, evitando pedras de gelo. Todas as anastómoses distais foram realizadas em primeiro lugar e depois é que todas as proximais foram realizadas no período de reaquecimento.

A desfibrilação do coração, quando necessária, foi feita à temperatura de $32^{\circ} \mathrm{C}$. Usamos luz fria frontal e lupa com aumento de $2,5 x$ ou $3,5 x$. Fios de marcapasso temporário foram colocados em menos de $5 \%$ dos casos.

$\mathrm{O}$ ar foi cuidadosamente retirado da aorta ascendente e das pontes, sendo, nestas, com auxilio de uma agulha de insulina.

\section{RESULTADOS}

Dos 260 pacientes operados, 28 faleceram nos primeiros 30 dias de pós-operatório, ou em conseqüência da operação (4,5\%). Em 19 dos procedimentos associados, $3(15 \%)$ pacientes faleceram, sendo que, nos 601 casos de revascularização do miocárdio isolada, a mortalidade foi de $4,3 \%$ (Tabela 1 ).

Em 1983 e 1984, a mortalidade foi de 0,9\%; em 1985 e 1986 , foi de $4,7 \%$ e, em 1987 , foi de $1 \%$ (Tabela 4).
TABELA 4

CIRURGIA DE REVASCULARIZAÇÃO DO MIOCARDIO 1983-1987

MORTALIDADE

\begin{tabular}{lccc}
\hline PERIODO & N. & MORTAL. & $\%$ \\
\hline $1983-1984$ & 106 & 1 & 0,9 \\
$1985-1986$ & 189 & 9 & 4,7 \\
1987 & 101 & 1 & 1,0 \\
\hline TOTAL & 396 & 11 & 2,7 \\
\hline
\end{tabular}

Os 28 óbitos ocorreram devido às causas apresentadas na Tabela 5 .

TABELA 5

REVASCULARIZAÇĀO DO MIOCARDIO

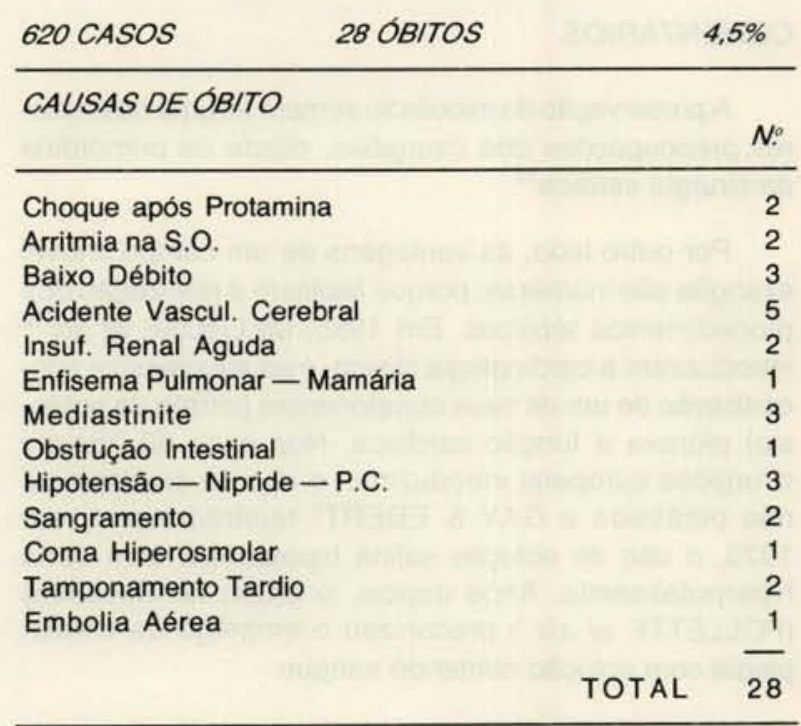

P.C. $=$ Parada cardíaca.

S.O. = Sala de operação.

A incidência de infarto pós-operatório foi em torno de $5 \%$. As causas de baixo débito no pós-operatório estiveram mais relacionadas com a função ventricular pré-operatória, com o grau de doença coronária e com a qualidade da anastomose, do que com o tempo de pinçamento total da aorta.

Arritmias ventriculares e supraventriculares, levando a baixo débito, aconteceram em 1 a $2 \%$ dos pacientes.

Dois pacientes apresentaram assistolia, imediatamente após a infusão de protamina, sendo um deles na reoperaçāo.

Em determinado período, sangramento maior do que o usual ( $200 \mathrm{ml} /$ hora) ocorreu em $9 \%$ dos pacientes, o que, recentemente, baixou para $3 \%$. 
JUCÁ, E. R.; CARVALHO Jr., W.; CIARLINI, C.; MESQUITA, F.; MONTE, L.; ALVES, M. G. A. - Cirurgia de revascularização do miocárdio sem o emprego de soluçōes cardioplégicas, balāo intra-aórtico, ou catéter de Swan-Ganz. Rev. Bras. Cir. Cardiovasc., 3(1): 21-28, 1988.

A incidência de cirurgia de coronária em relação à cirurgia cardíaca em geral foi de $33,8 \%$, em 1984 e 1985, e passou para $57,7 \%$, em 1987 (Tabela 6).

TABELA 6

INCIDEANCIA DE REVASCULARIZACCÃO DO MIOCÁRDIO EM RELAÇAOO A CIRURGIA CARDIACA

\begin{tabular}{cccc}
\hline ANO & C.C. & R.M. & $\%$ \\
\hline & & & \\
$1983-1984$ & 313 & 106 & 33,8 \\
1987 & 175 & 101 & 57,7 \\
\hline
\end{tabular}

R.M. = Revascularização do miocárdio.

C.C. $=$ Cirurgia cardiaca.

\section{COMENTÁRIOS}

A preservação do miocárdio sempre foi uma das maiores preocupaçōes dos cirurgiōes, desde os primórdios da cirurgia cardica $^{10}$.

Por outro lado, as vantagens de um campo imóvel exangüe são inúmeras, porque facilitam a realização dos procedimentos técnicos. Em 1955, MELROSE et alii ${ }^{12}$ introduziram a cardioplegia clínica, mas a excessiva concentração de um de seus componentes (citrato de potássio) piorava a função cardíaca. Nos anos 60 , alguns cirurgiōes europeus introduziram o uso de cardioplegia não potássica e GAY \& EBERT ${ }^{8}$ reintroduziram, em 1973 , o uso de solução salina hipotérmica com certa hiperpotassemia. Anos depois, o grupo de Buckberg (FOLLETTE et alii $^{7}$ ) preconizou o emprego da cardioplegia com solução contendo sangue.

Inúmeros trabalhos demonstram a eficácia do emprego de soluçōes cardioplégicas ${ }^{1,11}$. Entretanto, todos conhecem casos de efeitos nocivos do emprego destas soluçōes.

Embora os objetivos de uma solução cardioplégica ideal sejam teoricamente conhecidos, na prática ainda não dispomos desta soluçāo" ${ }^{11}$.

Os seguintes itens ainda são tópicos de controvérsia: cristalóide ou sangüinea; temperatura ótima para proteção máxima; temperatura corporal durante a cardioplegia; manutenção da parada diastólica; osmolaridade; quantidade e pressão da infusão; via anterógrada ou retrógrada pelo seio coronário; composiçăo de $\mathrm{K}^{+}$e do cálcio; aditivos com procaína, esteróides, manitol, nifedipina, verapamil, nitroglicerina; canulaçāo das duas veias cavas; edema de reperfusão; uso de glutamato e da cardioplegia morna, recentemente proposto por Buckberg. Quanto mais se lê sobre o assunto, maiores questōes surgem na mente $6,7,11$.
Por outro lado, há muitos anos que SHUMWAY \& LOWER $^{13}$ demonstraram que a hipotermia tópica protege o miocárdio por longos periodos de anóxia. A procura de órgãos à distância, para o transplante cardiaco, também demonstra a preservação através de hipotermia tópica.

Muitos cirurgiōes, no passado, e alguns, ainda, no presente, como JATENE et alii ${ }^{9}$; BRENOWITZ et alii ${ }^{4}$ e BONCHEK \& BURLIGAME ${ }^{3}$, demonstraram excelentes resultados com a técnica da reperfusão intermitente. Se o edema da reperfusāo fosse clinicamente tão comum, cirurgiōes como os do grupo de Jatene, que fazem as anastomoses distais e proximais com pinçamento ( 3 pontes: 6 pinçamentos) não obteriam tão bons resultados cirúrgicos.

Recentemente, BRENOWITZ et alii ${ }^{4}$, do grupo de W. D. Johnson, mostraram que, apesar de longos periodos de perfusāo e de pinçamento da aorta, bons resultados foram obtidos em casos de endarterectomia extensa. Estes autores preconizam 25 minutos de pinçamento, com 5 minutos de reperfusāo e, em experiência de 144 pacientes, com endarterectomia de 3 vasos, com tempo total de pinçamento de 195 minutos, as complicaçōes foram mínimas. Na nossa experiência, os tempos médios por pinçamento variaram de 15,52 para 20,56 minutos. Entretanto, uma anastomose distal leva, em média, 12 minutos. O nosso tempo médio total de pinçamento foi de 31,51 minutos $\pm 11,86$. Portanto, os nossos tempos de parada isquêmica sāo mais curtos do que os de BRENOWITZ et alii ${ }^{4}$, porém mais longos do que os de BONCHECK \& BURLIGAME ${ }^{3}$.

Estes últimos autores publicaram, recentemente, uma experiência com 500 casos operados com pinçamento intermitente, com $1 \%$ de mortalidade, e o tempo médio de isquemia foi 7,65 minutos por ponte, à temperatura corporal de $30^{\circ} \mathrm{C}$.

Os nossos resultados com pinçamento intermitente, embora não tão brilhantes como os de BRENOWITZ et alii ${ }^{4}$, JATENE et alii ${ }^{9}$, e BONCHECK \& BURLIGAME ${ }^{3}$, são paralelos a eles e nos induzem a manter o método.

Somos cônscios de algumas desvantagens do pinçamento intermitente: é mais trabalhoso, a quantidade de sangue aspirado é maior, o coração não fica tão flácido, há maior traumatismo da aorta ascendente. Entretanto, no que concerne à preservação do miocárdio, é muito bom, conforme atestam os resultados acima demonstrados e a função ventricular conservada de inúmeros pacientes operados e que, depois de alguns anos, foram reestudados com ventriculografia.

Nossa experiência com o pinçamento intermitente na cirurgia da valva mitral e da tétrade de Fallot nos convence, ainda mais, da aceitável preservaçăo do miocárdio que tal técnica propicia. Aliás, Dudley Johnson afirma que a sua técnica de endarterectomia longa da 
JUCÁ, E. R.; CARVALHO Jr., W.; CIARLINI, C.; MESQUITA, F.; MONTE, L.; ALVES, M. G. A. - Cirurgia de revascularização do miocárdio sem o emprego de soluçōes cardioplégicas, balão intra-aórtico, ou catéter de Swan-Ganz. Rev. Bras. Cir. Cardiovasc., 3(1): 21-28, 1988.

descendente anterior é otimizada pela perfusão intermitente.

A medida da pressão do átrio esquerdo e/ou do tronco pulmonar é um ótimo indicador do desempenho do ventrículo esquerdo. Alguns cirurgiōes usam-na de rotina no pós-operatório, diretamente, ou indiretamente, pelo catéter de Swan-Ganz.

Ambos apresentam alguns inconvenientes e, mesmo, complicações, sem falar no preço elevado do catéter de Swan-Ganz.

Usamos, de rotina, a pressāo do átrio esquerdo na saída da perfusão extracorpórea; quando somada a outros parâmetros, apresenta-se como bom guia para suporte inotrópico, ou volêmico.

Como a medição direta apresenta alguns riscos (trombose, embolia aérea e disfunção da válva mitral) e o catéter de Swan-Ganz é oneroso, não usamos o método, no pós-operatório, nessa experiência.
O suporte circulatório mecânico, temporário e eficaz, é de grande utilidade no pré e, principalmente, no pós-operatório de doentes graves. Até o momento, o balão intraaórtico (BIA) tem sido o mais usado. As reais indicaçōes do seu emprego são discutíveis.

Foi o BIA superusado em algumas Instituições?

Quando, ao suporte inotrópico, deve ser associado o suporte mecânico? o número de complicações por cento, que não é pequeno, torna difícil a análise risco/benefício, já que, em casos isolados, o seu papel pode ser espetacular. Por não dispormos de BIA, na Casa de Saúde São Raimundo, em nenhum dos pacientes foi ele usado.

Em suma, a técnica de cardioplegia com soluções geladas hipercalêmicas é a mais usada, atualmente, pela maioria dos cirurgiōes. Porém, a nossa experiência aqui relatada, bem como a de outos autores atestam que o pinçamento e a reperfusão intermitente, associados à hipotermia tópica e corporal, são um aceitável método de preservação do miocárdio, na cirurgia de revascularizaçāo.

RBCCV 44205-45

JUCÁ, E. R.; CARVALHO Jr., W.; CIARLINI, C.; MESQUITA, F.; MONTE, L.; ALVES, M. G. A. - Myocardial revascularization without cardioplegia, intra-aortic baloon or Swan-Ganz catheter. Rev. Bras. Cir. Cardiovasc., 3(1): 21-28, 1988.

ABSTRACT: From 1974 to December 1987,620 patients were submitted to myocardial revascularization, with 28 deaths, $4.5 \%$ mortality. Nineteen patients received associated procedures: 14 ventricular aneurysmectomy and 5 valvular prosthesis. The method of myocardial protection was intermitent clamping of the aorta, topical and body hypothermia of $30^{\circ} \mathrm{C}$. From this experience we conclude that this method offers a satisfactory myocardial preservation.

DESCRIPTORS: myocardial revascularisation, surgery; myocardial preservation.

\section{REFERÊNCIAS BIBLIOGRÁFICAS}

1 ADAPPA, M. G.; JACOBSON, L. B.; HETZER, R.; HILL, J. D.; KAMM, B.; KERTH, W. J. - Cold hyperkalemic cardiac arrest versus intermittent aortic cross-clamping and topical hypothermia for coronary bypass surgery. J. Thorac. Cardiovas. Surg., 75(2): 171-178, 1978.

2 AKINS, C. W. - Noncardioplegic myocardial preservation for coronary revascularization. J. Thorac Cardiovas. Surg., 88(2): 174-181, 1984.

3 BONCHEK, L. I. \& BURLINGAME, M. W. - Coronary artery bypass without cardioplegia. J. Thorac. Cardiovasc. Surg., 93(2): 261-267, 1987.

4 BRENOWITZ, J. B.; KAYSER, K. L.; JOHNSON, W. D. - Results of coronary artery endarterectomy and reconstruction. J. Thorac. Cardiovasc. Surg., 95(1): 1-10, 1988.
5 COOLEY, D. A.; REUL, G.; WUKASH, D. C. - Ischemic contracture of the heart: stone heart. Am. J. Cardiol., 29(4): 575-577, 1972.

6 DAVIES, A. L.; JUCÁ, E. R.; AUSTEN, W. G. - Left ventricular function in steroid pre-treated dogs following total cardiopulmonary by-pass. J. Cardiovas. Surg. (Torino) 9(3): 273-277, 1968.

7 FOLLETTE, D. M.; MULDER, D. G.; MALONEY Jr., J. V.; BUCKBERG, G. D. - Advantages of blood cardioplegia over continuous coronary perfusion or intermittent ischemic: experimental and clinical study. $J$. Thorac. Cardiovas. Surg., 76(5): 604-619, 1978.

8 GAY, W. A. \& EBERT, P. A. - Functional, metabolic and morphologic effects of potassium induced cardioplegia. Surgery, 74(2): 284-290, 1973. 
JUCÁ, E. R.; CARVALHO Jr., W.; CIARLINI, C.; MESQUITA, F.; MONTE, L.; ALVES, M. G. A. - Cirurgia de revascularização do miocárdio sem o emprego de soluçōes cardioplégicas, balāo intra-aórtico, ou catéter de Swan-Ganz. Rev. Bras. Cir. Cardiovasc., 3(1): 21-28, 1988.

9 JATENE, F. B.; FERREIRA, H. P.; AULER Jr., J. O. C.; OLIVEIRA, S. A.; RAMIRES, A. F; VERGINELLI, G.; PILEGGI, F. J. C.; JATENE, A. D. - Estudo comparativo da cardioplegia e do clampeamento intermitente em cirurgia de revascularização do miocárdio. Arq. Bras. Cardiol., 43(Supl. 1): 90, 1984 (Resumo).

10 KIRKLIN, J. W.; CONTI, V. R.; BLACKSTONE, E. H. Prevention of myocardial damage during cardiac operation. N. Engl. J. Med., 301(3): 135-141, 1979.

11 LAZAR, J. L. \& ROBERTS, A. J. - Recent advances in cardio-pulmonary by-pass and the clinical application of myocardial protection. Surg. Clin. N. Am., 63(3): 455-476, 1985.

12 MELROSE, D. G.; DREYER, B.; NENTALL, H. H.; BAKER, J. B. E. - Elective cardiac arrest. Lancet 2(6879): 21-22, 1955.

13 SHUMWAY, N. E. \& LOWER, R. R. - Topical cardiac hypothermia for extended periods of anoxic arrest. Surg. Forum, 10: 563-566, 1959.

\section{Discussão}

\section{DR. SEBASTIÃO RABELLO Belo Horizonte, M. G.}

Agradeço à Comissāo, pelo privilégio de comentar o brilhante trabalho do Dr. Jucá, e queria dizer o seguinte: em nossa experiênca, no Hospital Vera Cruz, em Belo Horizonte, trabalhamos, durante muitos anos, de uma maneira bastante simples, inclusive levados pelo espírito que o Dr. Cooley transmitiu a todos, de que o clampeamento da aorta, até 50 ou 55 minutos, era perfeitamente satisfatório e a recuperação miocárdica era mantida. Passaram-se muitos anos, para que se viesse a saber que esses clampeamentos prolongados, realmente, induziam a uma fibrose miocárdica, com prejuizo grande na recuperação final desse paciente. Baseado nisto, em parte, e na necessidade de dispormos de mais tempo para a execução de diversos procedimentos cirúrgicos, nós nos interessamos e passamos a praticar a cardioplegia da forma como veio sendo preconizada, utilizando, principalmente Saint Thomas e Saint Thomas modificado. Ainda, no nosso Serviço, temos um anestesista que estuda, constantemente, o problema e que tem acompanhado de perto a técnica de Buckberg e tem procurado adaptar, às nossas condiçōes, o uso dessa cardioplegia, que é uma carioplegia realmente complexa, difícil de ser realizada em nosso meio. No que diz respeito à cirurgia de revascularização do miocárdio, a impressão que, pessoalmente, tenho é a de que, com aqueles cirurgiōes realmente brilhantes, como o Dr. Regis, que são capazes de fazer uma anastomose bem feita, sem problemas algum, num tempo de 8,9,10 minutos, ou menos, o clampeamento intermitente permite a realização de cirurgias muito bem feitas, num prazo de oclusão dè aorta relativamente curto e com uma recuperação muito boa. Os números que ele apresentou, como resultado de seu trabalho, mostram que sāo tāo bons quanto os melhores do resto do mundo e acho que merece ser parabenizado, por esse tipo de resultado. Eu perguntaria ao Dr. Jucá se, no Hospital onde executou este trabalho, são examinados pacientes em que a cirurgia tenha que ser feita em situação muito crítica, em estado de emergência, em edema agudo de pulmặo e em que, sabidamente, o resultado é mais sério. Perguntaria, também, se, nas cirurgias não coronárias, em que o clampeamento da aorta é necessariamente prolongado, ele usa a cardioplegia, e a qual tipo daria preferência, no momento. Nós introduzimos, em nosso Serviço, SwanGanz,que ele comentá que não usou e nos pareceu de grande utilidade, em pacientes com baixo débito na saída de circulação extracorpórea. Eu agradeço, novamente, à Comissão e parabenizo o Dr. Jucá, pelo trabaIho.

\section{DR. RONALD PEIXOTO Campos, RJ}

Eu agradeço à Comissão Organizadora, pelo privilégio de comentar este trabalho, do Dr. Jucá. Em 1986, nós apresentamos, nos Arquivos Brasileiros de Cardiologia, os nosso primeiros casos operados, de 1973 a 1976, em que usamos, em grande parte deles, cardioplegia. O resultado dessa revascularização do miocárdio era de uma mortalidade de $6 \%$. Nessa mesma época, a nossa mortalidade de valvopatia aórtica e mitral era de $12 \%$, bem como dos congênitos. Recentemente, (slide) em agosto de 1987, fizemos uma revisão dos nossos pacientes e verificamos que a mortalidade era, exatamente, a mesma de 11 ou 12 anos atrás, usando cardioplegia, mas que, nos últimos 80 pacientes operados por valvopatia e congênitos, a mortalidade tinha caído abaixo de $2 \%$. Nós seguimos um protocolo bastante rígido, nessas valvopatias e nos congênitos, e resolvemos adotar o mesmo protocolo, um pouco modificado, nos pacientes coronarianos, a partir do ano passado. (Slide) Nós fazemos anastomose safena-aorta, antes da perfusão, exceto com as marginais, com o intuito de diminuir o tempo de circulação extracorpórea. (Slide). A preservação do miocárdio é feita com hipotermia sistêmica moderada, às vezes, com um pouquinho de soro no pericárdio (slide) e, principalmente, por cardioplegia, as primeiras cristalóides e as outras são sangüineas. A partir de 1987, resolvemos dar doses maiores e passamos $1000 \mathrm{cc}$ cada vez (slide) e repetimos, a cada 15 minutos; então, damos, primeiro, a solução cristalóide e as outras sangüíneas, (slide) através da aorta. Em alguns casos, passamos, também, através do átrio direito (slide), segundo os preceitos do Dr. Carpentier, (slide) sempre seguindo o conceito do Dr. Buckberg, de colocar o coração esvasiado, (slide) o que fazemos através da cânula atrioventricular 
JUCÁ, E. R.; CARVALHO Jr., W.; CIARLINI, C.; MESQUITA, F.; MONTE, L.; ALVES, M. G. A. - Cirurgia de revascularização do miocárdio sem o emprego de soluções cardioplégicas, balão intra-aórtico, ou catéter de Swan-Ganz. Rev. Bras. Cir. Cardiovasc., 3(1): 21-28, 1988.

esquerda e aspiração da aorta, quando estamos realizando as anastomoses distais. (Slide) Quando completamos as anastomoses e vamos fazer a reperfusão do coração, fazemos uma anticompressão total no ventrículo. Também ficamos muito impressionados com o trabalho do Dr. Buckberg, mostrando as lesōes de reperfusão. Por isso, fazemos uma solução aquecida, sangüinea, uma reperfusão controlada, colocando 150 a 200 $\mathrm{ml}$ por minuto, mantendo uma pressão de aorta de 50 $\mathrm{mmHg}$ e passamos de 1000 a $2000 \mathrm{cc}$ dessa solução cardioplégica aquecida e sangüínea, (slide) e, quando desclampeamos a aorta, o fazemos gentilmente. Quanto ao resultado, o número é pequeno, mas a nossa mortalidade baixou significantemente, porém não podemos tirar conclusão disto; sabemos, pelo menos, e espero, é que consigamos a mesma mortalidade da revascularização que nós temos em pacientes congênitos e valvares. $\mathrm{Pa}$ rabenizamos o Dr Jucá, pelo excelente trabalho e excelentes resultados e gostaríamos de colocar duas questões. É que ele explicasse melhor sobre as lesōes de reperfusão, pois estamos preocupados com isto. $\mathrm{O} \mathrm{Dr}$. Buckberg escreveu muito sobre isto, e usa o clampeamento intermitente da aorta. A segunda pergunta é se - Dr. Jucá usa a solução cardioplégica, ou cardioplegia, em alguns casos, e em que casos ele a usaria. Muito obrigado.

\section{DR. RONALDO FONTES São Paulo, SP}

Quero cumprimentar o Dr. Regis, pelos resultados apresentados com mortalidade de 2,7\%, nesses últimos anos. Gostaria de fazer alguns comentários. No Congresso da SBCCV de 1986, como o Dr. Regis mesmo citou, foi apresentado, pelo Dr. Fábio Jatene, um trabalho sobre o estudo de 2 grupos de pacientes com clampeamento intermitente e solução cardioplégica. Um estudo bem feito, estatisticamente correto, e ele demonstrou nāo haver diferença de resultados entre os 2 métodos de proteção miocárdica. Em relação a um dos tópicos do trabalho do Dr. Regis, que é o emprego de soluçāo cardioplégica, tenho a impressão de que estamos plenamente de acordo. Quanto ao uso de balāo intra-aórtico e catéter de Swan-Ganz, nós temos pontos que devemos considerar. O objetivo de seu trabalho, como está escrito no mesmo, seria não ser contra tais adjuntos, mas mostrar uma experiência sem o uso deles, para servir como uma linha de base, para comparaçōes entre outros grupos. Dr. Regis apenas conclui que o clampeamento intermitente, associado à hipotermia, é um método aceitável de preservação miocárdica. Eu estaria esperando um pouco mais de informaçōes, quanto ao não uso do balăo intra-aórtico e o catéter de Swan-Ganz, por dois motivos simples: porque o senhor teve $10 \%$ de complicação do baixo débito cardiaco na série e, em nenhum deles, o senhor usou o balão intra-aórtico e nós já sabemos que é um arsenal terapêutico que pode ser utilizado para tratar esses doentes. Gostaria de saber se o senhor fez isto deliberadamente, ou por falta de acesso a esses equipamentos. Obrigado.

\section{DR. CLÁUDIO SALLES \\ Belo Horizonte, MG}

Eu queria fazer um pequeno comentário sobre o uso do catéter de Swan-Ganz. Foi feito um estudo multicêntrico, em Boston, que foi publicado no final do ano passado, na revista Chest: trata-se de um estudo de um grande número de pacientes, e se verificou que as complicações com o uso do catéter de Swan-Ganz são maiores do que os benefícios que este pode trazer para o tratamento e, analisando grupos de pacientes, um em que esse tipo de catéter foi utilizado e outro em que não foi utilizado, verificou-se que a mortalidade era maior no grupo de pacientes em que foi utilizado. Este assunto tem causado muita discussão. Não sei, exatamente, se, nos Estados Unidos e em Belo Horizonte, no Hospital Felício Roxo, tinha-se essa impressão, de que os pacientes com Swan-Ganz complicavam mais, particularmente aqueles com infarto do miocárdio complicado e, a partir desse estudo, passou-se a não utilizar mais esse tipo de catéter.

\section{DR. JUCÁ}

(Encerrando)

Queria agradecer ao Dr. Sebastião os seus comentários e devo dizer que a nossa cirurgia é lenta; levamos 12 minutos por anastomose; nós somos lentos, principalmente em cirurgia de coronária, usamos lupa, luz, não ficamos com medo, quando demora a soltar a aorta, isto não, porque vimos as experiências de Shumway com hipotermia tópica. Hoje, com a procura do coração à distância, 3 horas só com a hipotermia, porque houve apenas uma cardioplegia, isto nos convence, ainda mais, de que o trabalho de Shumway estava correto. A nossa cirurgia é lenta. $O$ edema da reperfusão, sobre que o Dr. Ronaldo falou, e também o Buckberg, que foi citado pelo $\mathrm{Dr}$. Sebastião e pelo $\mathrm{Dr}$. Ronaldo, devo dizer que leio todos os trabalhos do Buckberg, mas, quando termino de ler, dá vontade de voltar para a cirurgia pulmonar, vesícula, hérnia, porque é uma coisa tão complicada, que, às vezes, estamos em outro planeta, embora não deixe de considerar que a contribuição dele é inestimável. Quando vejo a experiência do grupo do Dr. Jatene, do Dr. Luiz Carlos Bento, Dr. Sérgio Almeida de Oliveira, que, em 3 anastomoses, fazem 6 clampeamentos, sem que esse edema da reperfusão ocorra, imagino que nāo é tão importante, clinicamente, quando o Dr. Buckberg mostra. Insisto em que não quero desmerecer o seu trabalho, mas são pessoas que estão fazendo uma pesquisa que é mais experimental do que clínica. Eu também fiz muita pesquisa em cachorro, no Massachusetts General Hospital e tenho a convicção de que o cachorro tem o edema da reperfusão muito maior do que o homem. Quanto aos comentários do Dr. Ronaldo 
JUCÁ, E. R.; CARVALHO Jr., W.; CIARLINI, C.; MESQUITA, F.; MONTE, L.; ALVES, M. G. A. - Cirurgia de revascularização do miocárdio sem o emprego de soluçōes cardioplégicas, balāo intra-aórtico, ou catéter de Swan-Ganz. Rev. Bras. Cirr. Cardiovasc., 3(1): 21-28, 1988.

Fontes, quero dizer que nós usamos, de rotina, a pressão do átrio esquerdo na sala de operação; na saída de perfusão, o paciente fica canulado com agulha $30 / 8$, equipo de soro entre a aorta e a cava superior, em todo paciente, seja ele de qualquer gravidade; entretanto, os trabalhos com catéter de Swan-Ganz demonstraram isto, que há uma morbidade com eles, inclusive alguns pacientes apresentavam hipóxia no pós-operatório, porque, no ancoramento, existe hemorragia no capilar pulmonar. Eu não usei o balão intra-aórtico na Casa de Saúde de São Raimundo, porque não o temos. Possuímos um balấo intra-aórtico no Hospital de Mecejana. Quando eu estava no Massachusetts General Hospital, fui um dos primeiros a passar balão intra-aórtico experimentalmente, em porco. Participei desse começo, mas também não tenho dúvidas de que há pacientes que escaparam por causa do balâo intra-aórtico, mas também vi pacientes que faleceram pelo balāo intra-aórtico e que, de outra maneira, uma perfusão um pouco mais prolongada, um suporte inotrópico, poderia ter evitado o balāo. Na questão risco-benefício e custo-benefício, que não cabe no tamanho deste trabalho, é que entra bem essa questão do balão intra-aórtico e a questão do catéter de SwanGanz, que sāo adjuntos muito importantes, mas, se você colocar em percentagem o risco-benefício e o custo-benefício, eu tenho algumas dúvidas sobre o emprego. No Hospital Messejana, nós temos um balão intra-aórtico, mas, na Casa de Saúde São Raimundo, não temos. Eram estas as consideraçōes e agradeço as palavras dos comentadores. Obrigado. 